\title{
ARTINIAN QUOTIENT RINGS OF GROUP RINGS
}

\author{
Dedicated to the memory of Hanna Neumann
}

\section{IAN HUGHES}

(Received February 6 1973)

Communicated by M. F. Newman

\section{Introduction}

Smith [6, Theorem 2.18] proved that if $A$ is a ring which has a right artinian right quotient ring and $G$ is a poly- (cyclic-or-finite) group, then the group ring $A G$ has a right artinian right quotient ring. We give here a different proof (and a generalization) of this result using methods developed by Jategaonkar $[3,4]$. Explicitly we prove

THEOREM. Let $A$ be a ring which has a right artinian right quotient ring, and let $G$ be a group which has a (transfinite) ascending normal series with each factor either finite or cyclic, but only a finite number of finite factors. Then $A G$ has a right artinian right quotient ring.

We refer to Kuroš [5, p. 173] for the definition of an ascending normal series. We note that in the case of Smith's Theorem the group ring $A G$ is always right noetherian (if $A$ is) whereas this is not necessarily the case in our theorem.

REMARK. The restriction that there be only a finite number of finite factors in the ascending normal series in $G$ is necessary, as we can see from the following example.

Let $G$ be a group which is the union of a strictly ascending sequence of finite subgroups $\left\{G_{i}, i=1,2, \cdots\right\}$ where $G_{i}$ is normal in $G_{i+1}$ for each $i$. Let $K$ be a field. For each $i$ let $\omega\left(G_{i}\right)$ be the right ideal of $K G$ generated by $\left\{g-1 ; g \in G_{i}\right)$. It is readily seen that $\omega\left(G_{i}\right)$ is the right annihilator of $r_{i}=\Sigma g\left(g \in G_{i}\right)$ for each $i$. It is easy to see that $\left\{\omega\left(G_{i}\right)\right\}$ is strictly ascending. We thus have a strictly ascending infinite sequence of right annihilators in $K G$.

Now suppose $K G$ has an artinian Quotient ring $Q$. Certainly $Q$ has the ascending chain condition on right annihilators and so any subring, in particular $K G$, has this property. This contradicts what we have shown in the previous paragraph. 
Notation: Let $R$ be a ring. We denote by $P(R)$ the prime radical of $R$ and by $R^{*}$ the ring $R / P(R)$. We denote by $Q(R)$ the right quotient ring of $R$ when it exists. If $\rho$ is an automorphism of $R$ we denote by $\rho^{*}$ the automorphism of $R^{*}$ induced by $\rho$. If $R$ is semi-simple artinian, we denote by $m(R)$ the number of minimal two-sided ideals of $R$.

Let $G$ be a group and $R$ be a ring with identity. Suppose $(\eta, \omega)$ is a factor set for $G$ over $R$ (cf, for example, Bovdi [2]). We denote by $R[G ; \eta, \omega)$ the group ring (or crossed product) of $G$ over $R$ with factor set $(\eta, \omega)$.

For $m$ a positive integer we denote by $I_{m}$ the set $\{1,2, \cdots, m\}$.

In the remainder of this paper quotient ring will mean right quotient ring and artinian will mean right artinian.

\section{Group rings of infinite cyclic groups}

Let $G$ be an infinite cyclic group with generator $x$, and $A$ a ring with identity. Suppose $\rho$ is an automorphism of $A$. We denote by $A[G, \rho]$ the set of finite sums $\sum x^{i} a_{i}$ where $i$ is an integer and $a_{i}$ is in $A$. We define addition in the usual way, and multiplication by assuming the distributive law and the rule $a x=x \rho(a)$ for all $a$ in $A$. It is straightforward to check that $A[G, \rho]$ is an associative ring with identity. We remark that $A[G, \rho]$ is very similar to a skew polynomial ring as defined in [3]; however in our case negative powers of $x$ occur. But our situation is somewhat more special in that $\rho$ is an automorphism of $A$ and not merely a monomorphism of $A$ into itself.

Let $Q$ be a semi-simple artinian ring, and suppose $\left\{f_{i} ; i \in I_{m}\right\}$ is a complete set of primitive central idempotents in $Q$. Let $\rho$ be an automorphism of $Q$. Then, clearly, $\rho\left(f_{i}\right)=f_{\pi(i)}$ where $\pi$ is a permutation of $I_{m}$. Let $\pi=\pi_{1} \pi_{2} \cdots \pi_{t}$ be the (unique) decomposition of $\pi$ into disjoint cycles (we write 1-cycles also). For each $j$ in $I_{t}$ let $g_{j}=\Sigma f_{i}\left(i \in \pi_{j}\right)$ where $i \in \pi_{j}$ denotes that $i$ appears in the cycle notation for $\pi_{j}$. We denote $\pi_{j}$ by $(j 1 j 2 \cdots j m(j))$. Thus $\pi_{j}$ is a cycle of length $m(j)$ and $m=\Sigma m(j)\left(j \in I_{t}\right)$. If $t=1$ we say that $Q$ is $\rho$-transitive.

Now $Q=\oplus Q f_{i}\left(i \in I_{m}\right)$; and clearly for each $j$ in $I_{t}$ there is a division ring $D_{j}$ and a positive integer $n(j)$ such that for each $i\left(i \in \pi_{j}\right)$ there is an isomorphism

$$
\theta_{i}: Q f_{i} \rightarrow M_{n(j)}\left(D_{j}\right) \text {. }
$$

Also, if we denote $\theta_{j 1} \rho^{m(j)} \theta_{j 1}^{-1} \mid D_{j}$ by $\phi_{j}$ then it is easy to see that $\phi_{j}$ is an automorphism of $D_{j}$. Also, for each $j$ in $I_{t}$, we have $\rho\left(g_{j}\right)=g_{j}$ and if we denote $\rho \mid Q g_{j}$ by $\rho_{j}$ then $\rho_{j}$ is an automorphism of $Q g_{j}$ such that $Q g_{j}$ is $\rho_{j}$-transitive We have

Proposition 1 . Let $G$ be an infinite cyclic group, $Q$ a semi-simple artinian ring and $\rho$ an automorphism of $Q$. Then there is an isomorphism

$$
\psi: Q[G, \rho] \rightarrow \oplus M_{m(j)}\left(M_{n(j)}\left(D_{j}\left[G, \phi_{j}\right]\right)\right) \quad(j \in I)
$$


such that for $q$ in $Q$, the $j$ th component of $\psi(q)$ is given by a diagonal $m(j) \times m(j)$ matrix, namely

$$
\psi(q)_{j}=\operatorname{diag}\left(\theta_{j 1}\left(q f_{j 1}\right), \theta_{j 2}\left(q f_{j 2}\right), \cdots, \theta_{j m(j)}\left(q f_{j m(j}\right)\right)
$$

for each $j$ in $I_{t}$, where the notation is as described in the previous two paragraphs.

We omit the proof since we use essentially the same arguments that are used $\mathrm{n}$ the proofs of Theorem 2.1(a), Lemma 3.1(a) and Lemma 3.2 of [3].

We can now prove

Proposition 2. Let $A$ be a ring which has an artinian quotient ring $Q$ and suppose $\rho$ is an automorphism of $A$. Let $G$ be an infinite cyclic group. Then

(a) $A[G, \rho]$ has an artinian quotient ring $R$.

(b) (i) $P(R)=P(Q) R$

(ii) $P(Q)^{k}=0 \rightarrow P(R)^{k}=0$ ( $k$ a positive integer.)

(c) $R^{\#}$ is the quotient ring of $Q^{\#}\left[G, \rho^{\#}\right]$

(d) $m\left(R^{\#}\right) \leqq m\left(Q^{\#}\right)$

(e) If $m\left(Q^{\#}\right)=m\left(R^{\#}\right)=m$ (say) then for each $j$ in $I_{m}$ there is a positive integer $n(j)$ and two division rings $D_{j}$ and $E_{j}$ where $D_{j}$ is contained in $E_{j}$ such that there is a commutative diagram

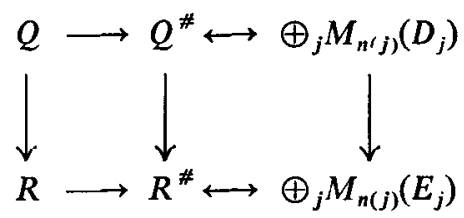

where the two double-headed arrows denote isomorphisms and for the rest, each homomorphism in the diagram is the obvious one.

Proof. We apply Proposition 1 to $Q^{\#}$ and use the same notation as we did in that proposition. By the usual argument using the division algorithm, for each $j$ we see that $D_{j}\left[G, \phi_{j}\right]$ is a principal right ideal domain and so has a quotient division ring which we denote by $E_{j}$. It follows from Proposition 1, that $Q^{\#}\left[G, \rho^{\#}\right]$ has a semi-simple artinian quotient ring which is isomorphic to $\oplus_{j} M_{m(i) n(j)}\left(E_{j}\right)\left(j \in I_{t}\right)$.

The proofs of (a), (b) (i) and (b) (ii) are now essentially the same as the proof of Theorem 3.1 of [4]. We therefore omit their proofs. Now (c) follows from (b) (i) using a routine argument; (d) follows from (c) since $m\left(R^{\#}\right)=t \leqq m=m\left(Q^{\#}\right)$.

We now prove (e). Since we are supposing $m\left(Q^{\#}\right)=m\left(R^{\#}\right)$, we see that $m(j)=1$ for each $j$. We may clearly assume that $\pi_{j}$ is the 1 -cycle $(j)$.

We let $Q^{\#} \leftrightarrow \oplus_{j} M_{n(j)}\left(D_{j}\right)\left(j \in I_{m}\right)$ be the isomorphism defined by the $\theta_{j}$ and let $R^{\#} \leftrightarrow \oplus_{j} M_{n(j)}\left(E_{j}\right)\left(j \in I_{m}\right)$ be the isomorphism defined by $\psi$ is Proposition 1 . 
We have thus obtained the required diagram. The commutativity of the left square follows from (b) (i) and that of the right square from the formula for $\psi(q)$ ( $q$ in $\left.Q^{\#}\right)$ given in Proposition 1. The proof is complete.

\section{Proof of theoren}

We assume the hypotheses of the theorem. A straight-forward argument shows that we may replace $A$ by its quotient ring. We may thus assume that $A$ is an artinian ring with identity.

Let the ascending normal series in $G$ be $\left\{G_{\alpha}, \alpha\right.$ an ordinal, $\left.G_{\Gamma}=G\right\}$. For each $\alpha<\Gamma$ we denote $G_{\alpha+1} / G_{\alpha}$ by $X_{\alpha}$. By assumption $X_{\alpha}$ is either finite or infinite cyclic. In the case where $X_{\alpha}$ is finite, we fix a set of coset representatives for $G_{\alpha}$ in $G_{\alpha+1}$ containing the identity of $G_{\alpha}$. It is easy to see that a factor set $\left(\eta_{\alpha}, \omega_{\alpha}\right)$ for $X_{\alpha}$ over $A G_{\alpha}$ is then determined and that the obvious map from $A G_{\alpha+1}$ to $A G_{\alpha}\left[X_{\alpha} ; \eta_{\alpha}, \omega_{\alpha}\right]$ is an isomorphism.

In the case where $X_{\alpha}$ is infinite cyclic, we choose as set of coset representatives of $G_{\alpha}$ in $G_{\alpha+1}$ the powers of an appropriate element $x$ in $G_{\alpha+1}$. The map $\rho_{\alpha}: G_{\alpha} \rightarrow G_{v}$ given by $\rho_{\alpha}(g)=x^{-1} g x$ for each $g$ in $G_{g}$ extends uniquely to an automorphism of $A G_{\alpha}$. It is easy to see that the obvious map from $A G_{\alpha+1}$ to $A G_{\alpha}\left[X_{\alpha}, \rho_{\alpha}\right]$ is an isomorphism.

For each $\alpha \leqq \Gamma$ we prove by transfinite induction on $\alpha$ that

(a) $A G_{\alpha}$ has an artinian quotient ring, which we denote by $Q_{\alpha}$.

(b) For $\gamma<\beta \leqq \alpha$ such that there are no finite jumps between $\gamma$ and $\beta$

(i) $m\left(Q_{\gamma}^{\#}\right) \geqq m\left(Q_{3}^{\#}\right)$

(ii) $P\left(Q_{\beta}\right)=P\left(Q_{\gamma}\right) Q_{\beta}$

(iii) $P\left(Q_{\gamma}\right)^{k}=0 \Rightarrow P\left(Q_{\beta}\right)^{k}=0$ ( $k$ a positive integer).

If in addition, $m\left(Q_{\gamma}^{\#}\right)=m\left(Q_{\beta}^{\#}\right)=m$ (say) then

(iv) For each $j$ in $I_{m}$ there is a positive integer $n(j)$ and division rings $D_{\gamma \cdot j}$ and $D_{\beta, j}$ such that $D_{\gamma, j} \subseteq D_{\beta, j}$ and such that there is a commutative diagram

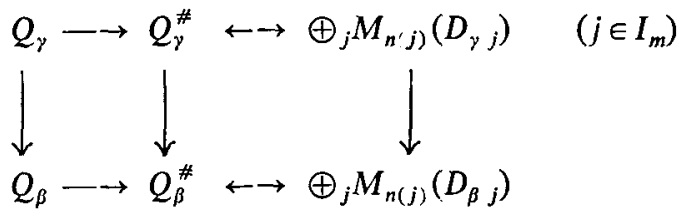

where the two double-headed arrows denote isomorphisms and for the rest each homomorphism in the diagram is the obvious one. 
We now prove these assertions. Suppose firstly that $\alpha$ is not a limit ordinal. If $X_{\alpha-1}$ is finite then as indicated above $A G_{\alpha}$ is isomorphic to

$$
A G_{\alpha-1}\left[X_{\alpha-1} ; \eta_{\alpha-1}, \omega_{\alpha-1}\right] \text {. }
$$

It is clear that the factor set $\left(\eta_{\alpha-1}, \omega_{\alpha-1}\right)$ can be extended to $Q_{\alpha-1}$. It is then straightforward to prove that $Q_{\alpha-1}\left[X_{\alpha-1} ; \eta_{\alpha-1}, \omega_{\alpha-1}\right]$ is the quotient ring of $A G_{\alpha}$ and that it is artinian. This proves (a) in this case. It is obvious that (b) is true also.

If $X_{\alpha-1}$ is infinite cyclic, then by the above $A G_{\alpha}$ is isomorphic to

$$
A G_{\alpha-1}\left[X_{\alpha-1}, \rho_{\alpha-1}\right]
$$

and both (a) and (b) are true by Proposition 2 .

We may thus assume that $\alpha$ is a limit ordinal. Since our ascending normal series in $G$ has only a finite number of finite factors, there is an ordinal $\delta<\alpha$ such that $G_{\delta}$ contains every finite jump preceeding $G_{\alpha}$. By applying (b) (i) of the inductive hypothesis it is clear, by enlarging $\delta$ if necessary, that we may assume that $m\left(Q_{\gamma}^{\#}\right)=m\left(Q_{\delta}^{\#}\right)=m$ (say) for all $\gamma$ satisfying $\delta \leqq \gamma<\alpha$.

We consider $Q_{\alpha}=$ inj limit $Q_{\gamma}(\delta \leqq \gamma<\alpha)$. It is easy to see that it is a quotient ring of $A G_{\alpha}$ (we use the fact that if an element is regular in $A G_{y}$ then it is regular in $A G_{\alpha}$ for $\gamma<\alpha$ ).

For each $j$ in $I_{m}$ we let $D_{\alpha j}=\operatorname{inj~limit~} D_{\gamma ;}(\delta \leqq \gamma<\alpha)$. We also let $Q_{\alpha}^{+}=$inj limit $Q_{\gamma}^{\#}(\delta \leqq \gamma<\alpha)$. Using (b)(iv) of the inductive hypothesis, it is straightforward to show that if in the diagram in (b) (iv) we replace $\beta$ by $\alpha$ and $Q_{\beta}^{\#}$ by $Q_{\alpha}^{+}$then the diagram is commutative, where the maps are the obvious ones. It follows that $Q_{a}^{+}$is semi-simple artinian.

If we can show that $Q_{\alpha}^{+}=Q_{\alpha}^{\#}$ then (b) follows easily. To prove (a), that is that $Q_{\alpha}$ is artinian, it suffices to show (cf [1], p. 71) that

(1) $Q_{\alpha}^{\#}$ is a semi-simple artinian ring

(2) $P\left(Q_{\alpha}\right)$ is a nilpotent

(3) $P\left(Q_{\alpha}\right)$ is finitely generated as a right ideal of $Q_{\alpha}$.

Using (b) (ii) of the inductive hypothesis it is easy to see that $I_{\alpha}=\operatorname{ker}\left(Q_{\alpha} \rightarrow Q_{\alpha}^{+}\right)$ is equal to $P\left(Q_{\gamma}\right) Q_{\alpha}$ for any $\gamma$ satisfying $\delta \leqq \gamma<\alpha$. For each such $\gamma$, we have that $Q_{\gamma}$ is an artinian ring with identity and so right noetherian. It follows that $I_{\alpha}$ is finitely generated as a right ideal of $Q_{\alpha}$. By applying (b) (iii) of the inductive hypothesis it is easy to see $I_{\alpha}$ is a nilpotent ideal and since $Q_{\alpha} / I_{\alpha}=Q_{\alpha}^{+}$is semisimple artinian it follows that $I_{\alpha}=P\left(Q_{\alpha}\right)$. Thus $Q_{\alpha}^{\#}=Q_{\alpha}^{+}$as required and (1), (2) and (3) are proved. Thus $Q_{\alpha}$ is artinian and the inductive proof is complete. The theorem follows by letting $\alpha=\Gamma$. 


\section{References}

[1] N. Bourbaki, Éléments de Mathématique, Algèbre chapitre 8, Modules et anneaux semisimples, (Hermann, Paris, 1958.)

[2] A. A. Bovdi, 'Crossed products of a semigroup and a ring', Dokl. Akad. Nauk SSSR 137 (1961), 1267-1268.

[3] A. V. Jategaonkar, 'Skew polynomial rings over semisimple rings', J. Algebra 19 (1971), 315-328.

[4] A. V. Jategaonkar, 'Skew polynomial rings over orders in artinian rings', J. Algebra 21 (1972), 51-59.

[5] A. Kurosh, The Theory of Groups, Vol. 2, (Chelsea, New York, 1956.)

[6] P. F. Smith, 'Quotient rings of group rings', J. London Math. Soc. (2) 3 (1971), 645-660.

Department of Mathematics

Queen's University

Kingston, Ontario, Canada 